Technical Note

\title{
Measurement of finger tapping performance using a smartphone application: a pilot study
}

\author{
Richard W Bohannon, DPT, EdD ${ }^{1)^{*}}$, Inga Wang, OTR, PhD ${ }^{2)}$ \\ 1) Physical Therapy Consultants: Fuquay-Varina, NC 27526 USA \\ 2) Department of Occupational Science and Technology, University of Wisconsin-Milwaukee, USA
}

\begin{abstract}
Purpose] Describe the measurement, reliability and validity of finger tapping repetitions recorded using a commercially available smartphone application (app). [Participants and Methods] We tested a convenience sample of 12 young right-handed participants who completed unilateral index finger tapping and peg board completion tasks with each hand. [Results] Measurement of finger tapping performance was practicable and was shown to be acceptably reliable and able to distinguish between performance of the dominant versus nondominant hand. Finger tapping was not correlated with pegboard performance. [Conclusion] A small sample of young adults showed that measures of finger tapping were easily obtained using a smartphone app. The measures demonstrated acceptable reliability and known groups validity. They, however, may not reflect performance at other measures of voluntary movement functions.

Key words: Hand, Dexterity, Measurement
\end{abstract}

(This article was submitted Feb. 8, 2021, and was accepted May 1, 2021)

\section{INTRODUCTION}

Control of voluntary movement is critical to independence in everyday activities ${ }^{1)}$. Numerous options exist for the clinical measurement of voluntary movement. Historically, however, its measurement has often involved use of instrumentation designed specifically for documenting the number of finger taps an individual can complete over a period of time such as 10 $\mathrm{sec}^{2}$. More recently, procedures using desk-top calculators ${ }^{3)}$ or computer keyboards ${ }^{4)}$ or mouses ${ }^{5)}$ have been proposed for measuring finger tapping performance. Currently, smartphone applications (apps) have also been employed for measuring finger tapping repetitions. The availability, portability, and simplicity of phone-based apps notwithstanding, our search of the literature revealed limited research addressing the measurement, reliability, and validity of the standard finger tapping test conducted using a smartphone app ${ }^{6,7)}$. The purpose of this pilot study, therefore was to describe the measurement, reliability and validity of finger tapping repetitions recorded using a commercially available smartphone app.

\section{PARTICIPANTS AND METHODS}

This project was approved by the Institutional Review Board of the University of Wisconsin at Milwaukee (No. 18.165). All participants signed an informed consent form. They were enrolled during the Spring of 2018. Based on the small number of left-hand dominant individuals and the finding that right-hand dominant individuals demonstrate more tapping asymmetry than left-hand dominant individuals ${ }^{4}$, the study was limited to individuals who self-identified their right upper limb as preferred for writing, using scissors, and playing sports. The required sample size, based on data from 7 volunteers, was found to be 4 based on an alpha of 0.05 , a power of 0.80 , and a mean between-side difference of 8.4 taps in 10 sec. We enrolled a convenience sample of 3 males and 9 females (mean age 24.6, range 22-33 years) to compensate for potential

*Corresponding author. Richard W Bohannon (E-mail: rbohannonpt@gmail.com)

(C2021 The Society of Physical Therapy Science. Published by IPEC Inc.

(c) (1) $\odot$ This is an open-access article distributed under the terms of the Creative Commons Attribution Non-Commercial No Deriva(CC) 
sample variability and/or attrition.

Our study required tested individuals to participate in 2 sessions -5 to 10 days apart. Demographics and tests of movement function (left and right finger tapping and grooved pegboard completion) were completed during the first session. Finger tapping tests were conducted again during the second session. The order of movement function tests was randomized. Finger tapping was tested using an I-phone app (SYBU Data Digital Finger Tapping Test (version 3.5)). The phone was placed on a table at the level of the xiphoid process directly in front of the seated participants. The forearm of their tested upper limb was completely supported on the table as was the heel of the hand and finger tips of all but the index finger (Fig. 1). On the command go, participants were to tap the phone screen as quickly as possible by flexing and extending the metacarpal phalangeal joint of the tested index finger. Stroking with the tested finger was not permitted. The tapping app counted the number of screen contacts (taps) accumulated in $10 \mathrm{sec}$. The mean number of taps for 3 trials and the best (highest) number of taps for 3 trials were summarized for each hand. The grooved pegboard was placed on a table in a position similar to that of the smart phone. On the command go, seated participants were to use one hand to retrieve grooved pegs from a dish and place them one-by-one as fast as possible in 25 slotted holes. The time to complete the test was documented. The test was performed twice and the average and best times determined. The test was then performed in the same way with the other hand.

Reliability of the tapping test was described in 2 ways. First the internal consistency of the mean and best finger tapping results for the left and right hands during the first session was determined using Cronbach's alpha. Test-retest reliability was described using a mixed model intraclass correlation coefficient (ICC) for the mean and best finger tapping repetitions between sessions. Validity was examined in 2 ways. First, known groups validity was examined by using a paired t-test to determine if the mean and best number of taps were significantly greater on the dominant right side than the nondominant left side during session 1. Convergent validity was examined by using Pearson correlation coefficients to describe the relationship of the mean and best number of taps with mean and best times for the grooved peg board test during session 1 .

\section{RESULTS}

Descriptive statistics for the 2 movement tests are presented in Table 1. Internal consistency was supported by Cronbach's alphas of 0.837 and 0.770 for the mean and best finger tapping performances of the first session. The test-retest reliability of the finger tapping test, as characterized using the ICC, was $0.729\left(\mathrm{CI}_{95 \%}=0.294\right.$ to 0.914$)$ for the left hand and 0.832 $\left(\mathrm{CI}_{95 \%}=0.517\right.$ to 0.949$)$ for the right hand. The paired t-test supported the known groups validity of the finger tap test with significantly more mean taps $(\mathrm{t}=6.34, \mathrm{p}<0.001)$ and best taps $(\mathrm{t}=4.61, \mathrm{p}=0.001)$ on the dominant than on the nondominant side. The Pearson correlations ( $\mathrm{r}=-0.238$ to 0.237 ) between finger tap and pegboard measures (whether mean or best) did not support the convergent validity of the tapping test with the pegboard test.

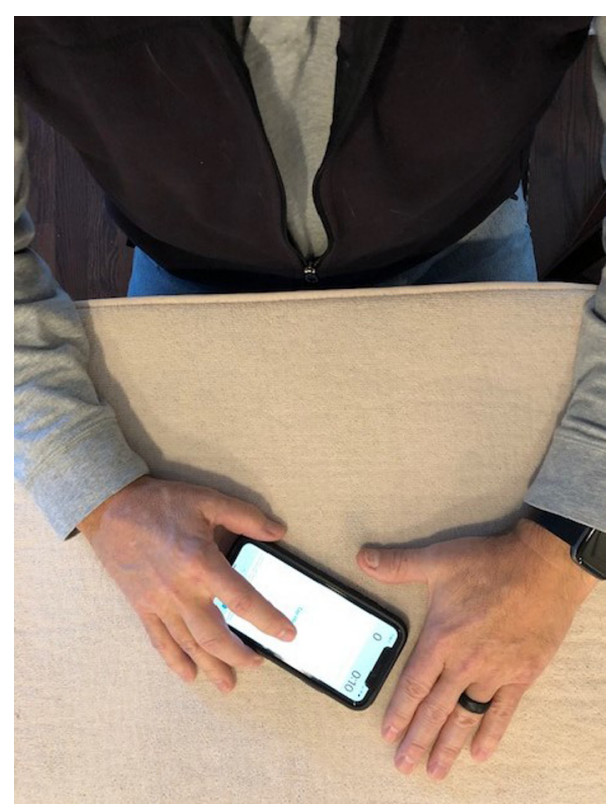

Fig. 1. Photograph showing position used to conduct finger tap test. 
Table 1. Descriptive statistics for the finger tapping test and the grooved pegboard test

\begin{tabular}{ccccccccc}
\hline \multirow{2}{*}{ Session } & \multicolumn{3}{c}{ Finger Tapping Test (Number in 10 sec) } & \multicolumn{3}{c}{ Grooved Pegboard Test (Time in sec) } \\
\cline { 2 - 8 } & \multicolumn{2}{c}{ Left hand } & \multicolumn{2}{c}{ Right hand } & \multicolumn{2}{c}{ Left hand } & \multicolumn{2}{c}{ Right hand } \\
\cline { 2 - 8 } & Mean & Best & Mean & Best & Mean & Best & Mean & Best \\
\hline 1 & $59.3[3.7]$ & $62.2[3.8]$ & $65.8[5.6]$ & $68.2[6.2]$ & $62.1[5.0]$ & $59.3[4.6]$ & $55.3[6.5]$ & $53.4[6.3]$ \\
2 & $60.8[4.9]$ & $63.6[4.9]$ & $67.0[8.7]$ & $69.1[8.6]$ & & & & \\
\end{tabular}

Numbers in cells are summaries of Mean (SD).

\section{DISCUSSION}

The purpose of this pilot study was to describe the measurement, reliability, and validity of a finger tapping test conducted using a smartphone app. No problems were encountered with the smartphone based measurement procedure. Whether this would be true using a different smartphone (eg, Samsung) and app (eg, CNS Tap Test) requires further research. All Cronbach's alpha and intraclass correlation coefficients were greater than 0.70 and thus provided support for the tapping test as adequately reliable. The known groups validity of the test was supported by better tapping performance on the dominant right side than the nondominant left side. Such performance differences between sides are consistent with those reported by others using different procedures ${ }^{2,5,8)}$. Convergent validity of the finger tapping test with the pegboard test was not supported as the correlations between measures obtained with the 2 tests were low. Perhaps a significant correlation should not be expected. Though both the tapping and peg tests might be categorized as reflective of movement function ${ }^{1)}$, they are quite different. Finger tapping requires repetition of the same uniplanar movement of a single finger. The peg test, to the contrary, requires the use and manipulation of the entire tested upper limb.

This study had several limitations. Primary among the limitations was the sample tested. It was limited in size and scope. That noted, the sample was adequate on the basis of power analysis. Moreover, the inclusion of only young right-hand dominant individuals produced results consistent with those reported by others using different procedures ${ }^{2}$.

\section{Conflict of interest}

None.

\section{REFERENCES}

1) International classification of functioning, disability and health. Geneva: World Health Organization, 2001, pp 94-95.

2) Mitrushina MN, Boone KB, Razani J, et al.: Handbook of normative data for neuropsychological assessment. New York: Oxford University Press, 1999, pp 419-443.

3) Worthington JA, De Souza LH: A simple measurement of speed of index finger movement. Clin Rehabil, 1989, 3: 117-123. [CrossRef]

4) Barut C, Kizıltan E, Gelir E, et al.: Advanced analysis of finger-tapping performance: a preliminary study. Balkan Med J, 2013, 30: 167-171. [Medline]

5) Hubel KA, Reed B, Yund EW, et al.: Computerized measures of finger tapping: effects of hand dominance, age, and sex. Percept Mot Skills, 2013 , 116: 929-952. [Medline] [CrossRef]

6) Lee CY, Kang SJ, Hong SK, et al.: A validation study of a smartphone -based finger tapping application for quantitative assessment of bradykinesia in Parkinson's disease. PLoS One, 2016, 11: e0158852. [Medline] [CrossRef]

7) Boukhvalova AK, Kowalczyk E, Harris T, et al.: Identifying and quantifying neurological disability via smart phone. Front Neurol, 2018, 9: 740. [Medline] [CrossRef]

8) Schmidt SL, Oliveira RM, Krahe TE, et al.: The effects of hand preference and gender on finger tapping performance asymmetry by the use of an infra-red light measurement device. Neuropsychologia, 2000, 38: 529-534. [Medline] [CrossRef] 